\title{
Four-way regulation of mosquito yolk protein precursor genes by juvenile hormone-, ecdysone-, nutrient-, and insulin-like peptide signaling pathways
}

\author{
Immo A. Hansen ${ }^{1,2,3}{ }^{*}$, Geoffrey M. Attardo ${ }^{4 *}$, Stacy D. Rodriguez ${ }^{1}$ and Lisa L. Drake ${ }^{1}$ \\ ${ }^{1}$ Department of Biology, New Mexico State University, Las Cruces, NM, USA \\ 2 Institute for Applied Biosciences, New Mexico State University, Las Cruces, NM, USA \\ ${ }^{3}$ Molecular Biology Program, New Mexico State University, Las Cruces, NM, USA \\ ${ }^{4}$ Department of Epidemiology of Microbial Disease, Yale School of Medicine, Yale University, New Haven, CT, USA
}

Edited by:

Xanthe Vafopoulou, York University,

Canada

Reviewed by:

Takashi Koyama, Instituto

Gulbenkian de Ciencia, Portugal

Shi-Hong Gu, National Museum of

Natural Science, Taiwan

\section{*Correspondence:}

Immo A. Hansen, Department of

Biology, New Mexico State

University, Foster Hall, 1200 South

Horseshoe, Las Cruces, NM 88003,

USA

e-mail:immoh@nmsu.edu:

Geoffrey M. Attardo, Department of Epidemiology of Microbial Disease

Yale School of Public Health, Yale

University, 60 College Street, New

Haven, CT 06517, USA

e-mail: geoffrey.attardo@yale.edu
Anautogenous mosquito females require a meal of vertebrate blood in order to initiate the production of yolk protein precursors by the fat body. Yolk protein precursor gene expression is tightly repressed in a state-of-arrest before blood meal-related signals activate it and expression levels rise rapidly. The best understood example of yolk protein precursor gene regulation is the vitellogenin-A gene $(\mathrm{vg})$ of the yellow fever mosquito Aedes aegypti. Vg-A is regulated by (1) juvenile hormone signaling, (2) the ecdysone-signaling cascade, (3) the nutrient sensitive target-of-rapamycin signaling pathway, and (4) the insulin-like peptide (ILP) signaling pathway. A plethora of new studies have refined our understanding of the regulation of yolk protein precursor genes since the last review on this topic in 2005 (Attardo et al., 2005). This review summarizes the role of these four signaling pathways in the regulation of $v g-A$ and focuses upon new findings regarding the interplay between them on an organismal level.

Keywords: mosquito, vitellogenesis, insulin, juvenile hormone, ecdysone, target of rapamycin, yolk proteins

\section{INTRODUCTION}

\section{MOSQUITO SPECIES USE TWO DIVERGENT REPRODUCTIVE STRATEGIES}

Mosquito species can be divided into two groups according to their reproductive strategy. Autogenous mosquitoes do not require an initial blood meal and produce their first batch of eggs relying solely on nutrients accumulated during their larval phase. Vitellogenesis, the production of yolk by the fat body for deposition in developing oocytes, begins a few hours after eclosion of autogenous adult females. Subsequent egg batches are dependent upon the energy and nutritional building blocks derived from vertebrate blood.

In contrast, anautogenous mosquitoes require an obligatory blood meal to produce their first batch of eggs. In these species, vitellogenesis is tightly repressed until blood meal-associated signals activate signaling cascades in involved organs and tissues. Most mosquito species fall into one of these two categories. However, there are exceptions to this rule. For example, Aedes atropalpus has both autogenous and anautogenous strains. Most of the human disease-transmitting species fall into the anautogenic category. The underlying reason for this is that the necessity for blood by anautogenous mosquitoes drives increased interaction between vector and host making them inherently better disease vectors. In addition, the fact that they feed earlier gives the parasites more time to finish the extrinsic part of their life cycle and become infective within the vector. Autogeny vs. anautogeny is discussed in more detail in Attardo et al. (2005).

\section{YOLK PROTEIN PRECURSOR PROTEINS ARE ESSENTIAL FOR MOSOUITO REPRODUCTION}

Insect oocytes are loaded with yolk proteins during the process of vitellogenesis. Yolk proteins provide the essential nutrients required for embryonic development. Mosquitoes produce yolk protein precursors (YPPs) exclusively in the fat body, the insect analog of the vertebrate liver. The YPPs are secreted into the hemolymph and taken up by the developing oocytes via receptor-mediated endocytosis (Sappington et al., 1996). Several yolk protein genes from Ae. aegypti have been independently cloned and characterized (Deitsch and Raikhel, 1993; Cho et al., 1999; Sun et al., 2000). The publication of the annotated Ae. aegypti genome sequence in 2007 (Nene et al., 2007) facilitated the identification of all potential yolk protein genes in silico. A detailed RNA-Seq comparison of genes differentially expressed by the Ae. aegypti fat body before and after a blood meal revealed that two genes encoding vitellogenic cathepsin $\mathrm{B}$, three genes encoding vitellogenins (vitellogenin A, B, C), and three genes encoding vitellogenic carboxy-peptidases are upregulated several hundredfold in the female fat body $24 \mathrm{~h}$ after a blood meal (Price et al., 2011). Together with three lesser expressed vitellin membrane proteins, yolk proteins account for more than a third of all messenger RNAs at this time point which represents the pinnacle of vitellogenesis. The abundance of YPP associated gene transcripts reflects the massive scale of protein synthesis the fat body performs during this process. 


\section{SIGNALING PATHWAYS IN THE FAT BODY}

Several signaling pathways regulate the transition of the fat body from the previtellogenic state-of-arrest to vitellogenic YPP synthesis. Our understanding of the interplay between these pathways has grown significantly in the last few years (see Figure 1). In this review we summarize the latest findings on each of the following topics: juvenile hormone, ecdysone, nutrient, and insulin-like peptide signaling pathways in mosquitoes, the roles they play in regulating vitellogenic gene expression in fat body trophocytes and the crosstalk that occurs between these signaling pathways on an organismal level.

\section{JUVENILE HORMONE \\ WHAT IS IT?}

Juvenile hormone $(\mathrm{JH})$ is a sesquiterpenoid that regulates insect development, reproduction and other processes in basically all insect species. Different groups of insects use different forms of JH, for instance mosquitoes use JH III (Clements, 1992).

\section{WHERE IS IT MADE?}

$\mathrm{JH}$ is synthesized by the corpora allata, a pair of specialized glands that are attached to the brain through the nervus corpora cardiaca and are in close proximity to the aorta.

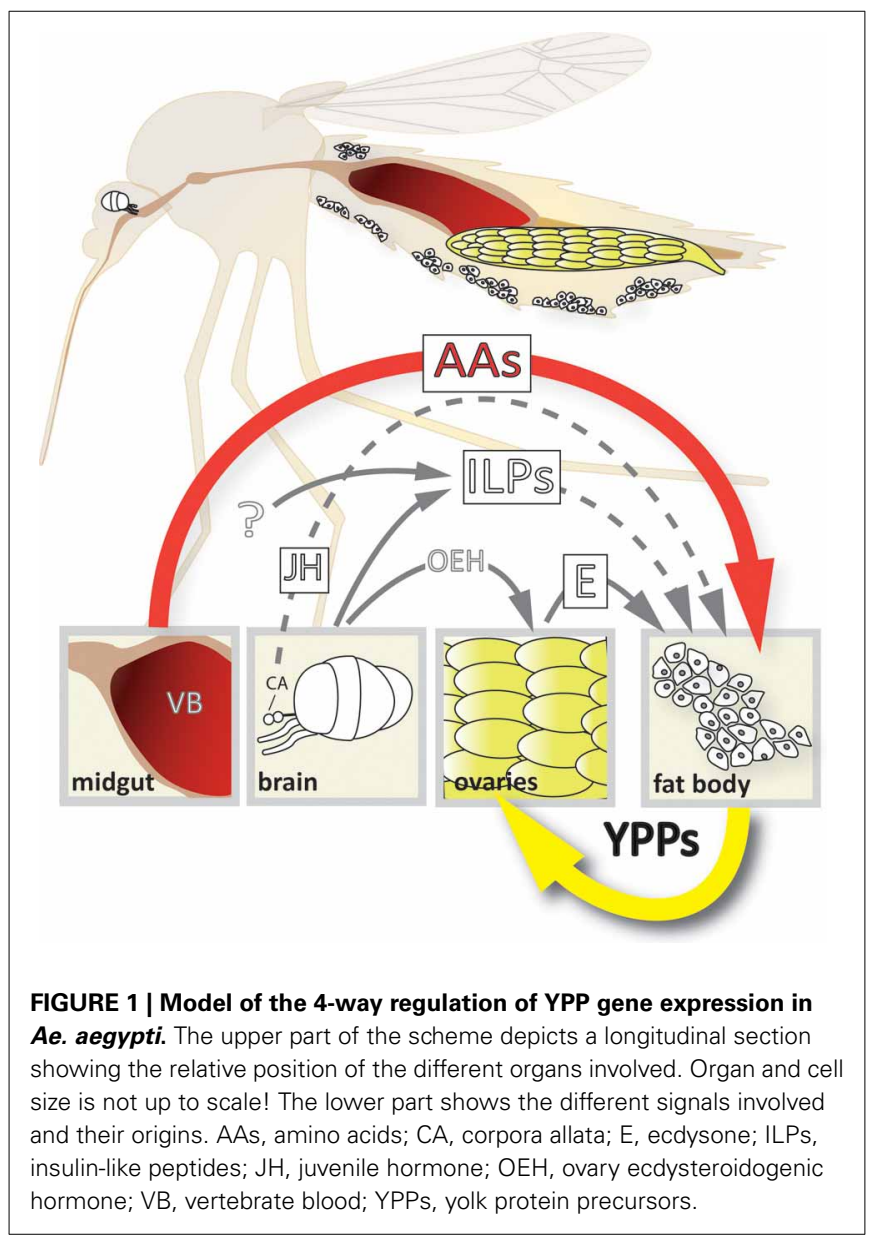

\section{HOW IS JH SYNTHESIS REGULATED?}

$\mathrm{JH}$ levels in the hemolymph and target tissues are thought to be regulated primarily by the rate of synthesis in the corpora allata. JH synthesis is controlled by peptide hormones that reach the glands through the hemolymph or by direct neural connections. Peptide hormones that stimulate $\mathrm{JH}$ synthesis are called allatotropins, those that inhibit $\mathrm{JH}$ synthesis are termed allatostatins (Gade et al., 1997). Signaling activity of JH is also regulated by proteins that facilitate its transport or breakdown in the hemolymph. Proteins promoting $\mathrm{JH}$ hydrolysis include $\mathrm{JH}$ esterase and JH epoxide hydrolases (Lassiter et al., 1994, 1995, 1996; Edgar et al., 2000; Bai et al., 2007), while JH-binding proteins facilitate the transport of this lipophilic molecule in the hemolymph (Prestwich et al., 1996). The rate of JH synthesis in newly eclosed female mosquitoes is in close correlation with their nutritional status (Noriega, 2004).

\section{MODE-OF-ACTION}

See Figure 2A-For several decades, efforts to identify a JHreceptor were unsuccessful. However, in recent years the understanding of JH's mode-of-action has grown significantly (Riddiford, 2012; Jindra et al., 2013). While there are still many open questions, there is growing evidence that the Methoprenetolerant (Met) protein (and in Drosophila also its paralog germcell-expressed) acts as intracellular JH-receptor. In complex with other proteins these receptors form active complexes that bind $\mathrm{JH}$-response elements in the DNA and regulate gene transcription (Li et al., 2011; Zou et al., 2013).

\section{HOW DOES JH REGULATE MOSOUITO YPP EXPRESSION?}

As its name suggests, one of the functions of JH during early postembryonic development is to prevent insect larvae from entering metamorphosis during the earlier larval molts. Another important role of $\mathrm{JH}$ is the regulation of insect reproduction. $\mathrm{JH}$ plays a central role in the regulation of YPP genes in mosquito females (Zou et al., 2013). After eclosion, females of anautogenous mosquito species enter a 3 day maturation period during which they do not blood-feed. Once this period is complete, the now "competent" females enter a state-of-arrest during which vitellogenic activity by the fat body is halted. The state-of-arrest is only broken when a blood meal is taken. Autogenous mosquitoes express YPP genes in the fat body earlier and without a blood meal stimulus; for example the vitellogenin-1 gene of Culex tarsalis is strongly expressed $24 \mathrm{~h}$ after eclosion (Provost-Javier et al., 2010). The ability or inability of an individual anautogenous mosquito to overcome the state-of-arrest and activate YPP gene expression is pre-established by its individual JH exposure history which in turn is correlated with the level of nutrients acquired during the larval phase (Noriega, 2004). Groups of Ae. aegypti larvae that are raised under severe nutritional restriction and overcrowded conditions give rise to significantly smaller adults compared to well-nourished under-crowded groups. Such smallsized Ae. aegypti females require more than one blood meal to complete vitellogenesis and develop a first batch of eggs (Shiao et al., 2008). This phenotype is due to the altered endocrinology of small mosquitoes; specifically these mosquitoes have lower $\mathrm{JH}$ levels during the 3 day maturation period which results in 


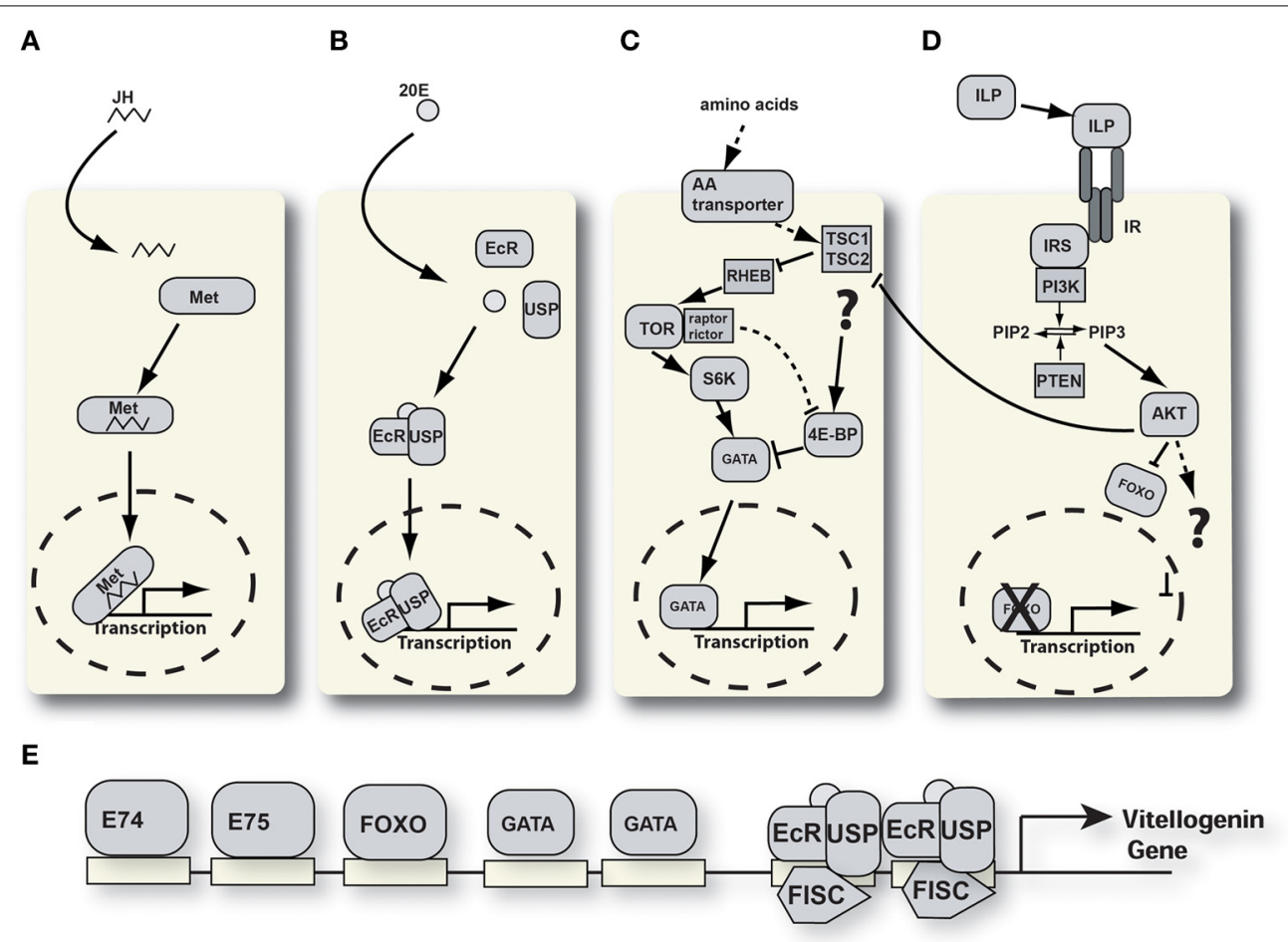

FIGURE 2 | Signaling pathways involved in YPP regulation. (A) Juvenile hormone signaling pathway. JH, juvenile hormone; Met, methoprene tolerant. (B) Ecdysone signaling pathway. 20E, 20 hydroxyecdysone; EcR, ecdysone receptor; USP, ultraspiracle. (C) Nutrient signaling pathway. 4E-BP, 4E-binding protein; TSC, tuberous sclerosis complex; RHEB, RAS homologue enriched in brain; S6K, S6 kinase; TOR, target of rapamycin. (D) Insulin-like peptide signaling pathway. AKT, protein kinase $\mathrm{B}$; $\mathrm{FOXO}$, forkhead box protein O; ILP, insulin-like peptide; IRS, insulin receptor substrate; PI3K, phosphati dylinositide 3-kinase; PIP2, phosphatidylinositol 4,5-bisphosphate; PIP3, phosphatidylinositol $(3,4,5)$-triphosphate; PTEN, phosphatidylinositol-3,4,5trisphosphate 3-phosphatase. (E) Schematic of the vg-A promoter with transcription factor binding sites. the delayed onset of vitellogenesis (Noriega, 2004). Treatment of small mosquitoes with $\mathrm{JH}$ immediately after eclosion results in recovery of the nutrient-signaling machinery (see Nutrient signaling) in the fat body, timely vitellogenesis and the successful development of a batch of eggs after only one blood meal.

Therefore, the role that $\mathrm{JH}$ plays in the regulation of YPP expression in the fat body is most likely a preparatory one. Managing $\mathrm{JH}$ levels is critical for mosquitoes to regulate and coordinate their nutrient reserves with their reproductive cycle. This hormonally mediated balance avoids nutrient shortages and optimizes egg production.

It must be emphasized that the effects of $\mathrm{JH}$ on vitellogenesis and egg development are in no way restricted to the mosquito fat body. For example, a study by Clifton and Noriega showed that $\mathrm{JH}$ levels determine the fate of individual ovarian follicles in Ae. aegypti thereby determining the final egg numbers that are produced in a given gonotropic cycle (Clifton and Noriega, 2012).

\section{ECDYSONE}

\section{WHAT IS IT?}

Ecdysteroids are insect steroid hormones which, like $\mathrm{JH}$, are key regulators of molting as well as reproduction in all holometabolous insects. Mosquitoes use the ecdysteroid ecdysone which is thought to be converted to its more bioactive form, 20 -hydroxyecdysone (20E), in the fat body and other peripheral tissues, as described in Drosophila (Raikhel et al., 2002; Gilbert, 2004; Rewitz et al., 2006).

\section{WHERE IS ECDYSONE MADE?}

During larval development ecdysone is synthesized by the prothoracic glands. However, these glands degenerate and disappear rapidly after adult emergence (Clements, 1992). In adult female mosquitoes ecdysone is produced by the epithelial cells of the ovarian follicle in response to blood meal-derived signals (Hagedorn, 1982, 1989; Raikhel et al., 1999).

\section{HOW IS ECDYSONE SYNTHESIS REGULATED?}

During larval development a peptide hormone, prothoracicotropic hormone (PTTH), is released from the brain and stimulates ecdysone synthesis in the prothoracic gland by activating the RAS/ERK pathway (Gilbert et al., 2002; Rewitz et al., 2009). In adult females a different peptide hormone, the ovary ecdysteroidogenic hormone $(\mathrm{OEH})$, substitutes the role of PTTH. $\mathrm{OEH}$ is produced in neurosecretory brain cells and stored in the corpora cardiaca (Brown and Cao, 2001). Upon blood feeding, stretch receptors within the midgut wall trigger a signal to the brain and $\mathrm{OEH}$ is released into the hemolymph of the mosquito (Hagedorn et al., 1975). OEH stimulates the follicle cells of the ovaries to synthesize and release ecdysone (Dhara et al., 2013). Four hours post-blood meal (PBM) ecdysone hemolymph titers 
are slightly elevated. Levels continue to rise reaching a peak at 18$24 \mathrm{~h} \mathrm{PBM}$ and decline afterwards (Hagedorn et al., 1975). OEH is a prominent regulator of ecdysone synthesis, but is by no means the only factor involved. Insulin-like peptide signaling also plays a role (see below).

\section{MODE-OF-ACTION}

See Figure 2B, 20E activates transcription of target genes by binding to a cytoplasmic receptor. The ecdysone receptor is a heterodimeric complex consisting of two proteins, the ecdysone receptor $(\mathrm{EcR})$ and ultraspiracle (USP), a retinoid $\mathrm{X}$ receptor (RXR) homolog (Kapitskaya et al., 1996; Hall and Thummel, 1998; Wang et al., 2000a, 2002). Upon binding 20E, the EcR/USP/20E receptor complex binds to ecdysone-response elements in the promoter region of target genes and activates transcription (Tran et al., 2001; Marquardt, 2005). Functional ecdysone-response elements are critical constituents of Ae. aegypti YPP gene promoters (Martin et al., 2001a). The ecdysone signal is subsequently amplified by a cascade of primary and secondary response genes that are activated by rising ecdysone levels (Spindler et al., 2009).

\section{HOW DOES 20E REGULATE YPP GENES?-PREVITELLOGENIC PHASE}

During the previtellogenic state-of-arrest YPP gene expression is tightly repressed by multiple mechanisms, several of them affecting the reception of ecdysone signals. Both, EcR, and USP proteins are expressed during this stage in the Ae. aegypti female fat body (Yao et al., 1992; Wang et al., 2000b). However, these proteins do not form a heterodimer capable of binding to ecdysone response elements during this period. One key factor inhibiting the ecdysone hormonal cascade is the competitive binding of the orphan nuclear receptor AHR38 to USP during previtellogenesis (Zhu et al., 2000; Marquardt, 2005). After a blood meal and in the presence of 20E, EcR displaces AHR38 and forms a heterodimer with USP. Sevenup (Svp), a mosquito homolog of chicken ovalbumin upstream transcription factor, acts as a transcriptional repressor by binding directly to AGGTCA repeats within the ecdysone response elements of the vg-1 promoter, thereby inhibiting the ecdysone responsiveness of these promoters (Miura et al., 2002).

\section{HOW DOES 20E REGULATE YPP GENES? - VITELLOGENESIS}

During the vitellogenic period, ecdysone is converted to $20 \mathrm{E}$ by hydroxylation within the cytoplasm of the fat body cells. It activates gene transcription within trophocytes directly by binding to the EcR/USP heterodimer. This complex then translocates into the nucleus and binds to ecdysone receptor response elements (Figure 2A) (Hall and Thummel, 1998; Wang et al., 1998). Aedes aegypti has two characterized $\mathrm{EcR}$ and USP isoforms (AaEcRa and $A a \mathrm{EcRb}$ AaUSPa and AaUSPb) (Kapitskaya et al., 1996; Wang et al., 2000a, 2002).

An orphan nuclear factor, $\beta$ FTZ-F1, mediates fat body competence for $20 \mathrm{E}$ responsiveness. $\beta \mathrm{FTZ}-\mathrm{F} 1$ is upregulated pre-and post-vitellogenesis and interacts with a p160/SRC type coactivator, FISC, to directly recruit EcR/USP/FISC to 20E promoters at the onset of the 20E signaling (Li et al., 2000; Raikhel et al., 2002; Zhu et al., 2006; Ou and King-Jones, 2013). Transcripts for another nuclear receptor, HR3, increase over the $24 \mathrm{~h}$ after a blood meal suggesting it also plays a role in $20 \mathrm{E}$ responsiveness (Kapitskaya et al., 2000). 20E activation of EcR/USP induces the early response genes, E74, E75, and Broad, which in turn, activate transcription of late target genes like $v g-1$ to amplify the hormonal signal (Raikhel et al., 2002; Sun et al., 2004; Chen et al., 2005).

Interestingly, $20 \mathrm{E}$ is also found in the male accessory glands of Anopheles gambiae mosquitoes and large amounts are transferred to females during mating. This suggests that $20 \mathrm{E}$ plays a role in modulating post-mating effects in females. However, a role for male derived $20 \mathrm{E}$ in YPP gene regulation has not yet been demonstrated in this mosquito (Pondeville et al., 2008).

\section{NUTRIENT SIGNALING \\ WHAT IS THE SIGNAL?}

Mosquitoes ingest vertebrate blood which functions as a rich source of amino acids. Amino acids function as building blocks for yolk protein precursor synthesis and are also used for energy production (Marquardt, 2004). The bulk of the protein contained within vertebrate blood is hemoglobin which is digested into free amino acids. The amino acids are transported via the hemolymph to the tissues of the female mosquito. After a blood meal, free amino acid levels in the mosquito hemolymph rise sharply as hemoglobin digestion in the mosquito midgut proceeds (Marquardt, 2004).

\section{MODE-OF-ACTION}

See Figure 2C The fat body monitors hemolymph amino acid levels via the target of rapamycin (TOR) signaling pathway which conducts the amino acid mediated signal to regulate YPP gene expression (Hansen et al., 2004). The key enzyme of this pathway, the TOR kinase, is a highly conserved protein that regulates protein translation in eukaryotic cells.

\section{HOW DOES THE TOR SIGNALING PATHWAY REGULATE YPPs?}

The exact mechanism eukaryotic cells utilize to sense hemolymph amino acid levels remains unknown. Amino acids are taken up by fat body trophocytes through a collection of specific amino acid transporter proteins that have different substrate specificities and may also work as receptors. The SLC7 family of cationic amino acid transporters play an important role in this process and are at the top of the nutrient signaling cascade in the fat body (Carpenter et al., 2012). RNAi-mediated gene knockdown of several of the SLC7-type transporters resulted in diminished TORsignaling, YPP expression, and egg production (Attardo et al., 2006; Carpenter et al., 2012). The small GTPase Rheb functions upstream of TOR and is an indispensable part of this pathway in the fat body (Roy and Raikhel, 2011). Several downstream components of the TOR pathway are also associated with nutrient signaling in the fat body (Figure 2B). A well characterized downstream player in the TOR pathways is the S6 Kinase. S6 kinase is directly phosphorylated by TOR (Hansen et al., 2005) and in turn activates the translation of a GATA transcription factor in the fat body (AaGATAa). During the state-of-arrest, YPP gene transcription is repressed by another GATA transcription factor (AaGATAr) that recognizes and binds to GATA-binding motifs upstream of the YPP gene region. Reception of a nutritional signal 
results in GATAr being displaced by GATAa upon the vg1 promoter. GATAa acts as a transcriptional activator which enhances YPP gene expression (Martin et al., 2001b; Attardo et al., 2003; Park et al., 2006). 4E-BP, a repressor of translation, is also a downstream target of TOR (Hay and Sonenberg, 2004). After a blood meal 4E-BP is hyper-phosphorylated in the mosquito fat body. However, treatment with the TOR inhibitor rapamycin does not block 4E-BP hyper-phosphorylation which indicates the involvement of other signaling pathways (Roy and Raikhel, 2012).

\section{INSULIN-LIKE PEPTIDE SIGNALING WHAT IS IT?}

Insulin-like peptides (ILPs) are an evolutionary conserved group of peptide hormones which are characterized by a conserved disulfide bond structure (De Meyts, 2004). ILP signaling plays a crucial role in immunity, reproduction and longevity of mosquitoes (Luckhart and Riehle, 2007). There are eight ILPs and one insulin receptor described in Ae. aegypti (Graf et al., 1997; Riehle et al., 2006).

\section{WHERE ARE ILPS EXPRESSED?}

ILPs and the insulin receptor are expressed in a variety of tissues in Ae. aegypti (Riehle and Brown, 2002; Riehle et al., 2006). Five ILPs are expressed in the brain and two are expressed in the head, thorax and abdomen of all life stages. These peptides assume a variety of possible roles including regulation of metabolic processes, cellular growth, lipid and glycogen processing, reproduction, and aging (Wu and Brown, 2006). Of the eight ILPs, ILP1, ILP3, and ILP 8 retain extremely conserved structures that are homologous to human insulin and relaxin 2 (Brown et al., 2008).

\section{ILPS RECEPTOR}

All known insulin receptors have tyrosine kinase activity. The first arthropod insulin receptor was characterized in D. melanogaster (Wu and Brown, 2006). The one known mosquito ILP receptor bears high homology to vertebrate insulin receptors and is comprised of two subunits, alpha and beta (Graf et al., 1997; Riehle and Brown, 1999). The alpha subunit contains the insulin binding domain while the beta subunit contains a tyrosine kinase domain which is responsible for the phosphorylation of downstream targets. Administration of human and porcine insulin to mosquitoes is capable of activating ILP signaling via their endogenous insulin receptor (Roy et al., 2007; Pakpour et al., 2012).

\section{MODE OF ACTION}

See Figure 2D Binding of the insulin receptor by ILPs activates a conserved signaling pathway as illustrated in Figure 2D (Wu and Brown, 2006). The phosphorylated insulin receptor substrate activates signaling via PI3K and other pathways. Downstream kinases phosphorylate effector proteins that regulate gene expression and other cellular processes.

\section{HOW DOES INSULIN REGULATE YPPS GENES?}

ILPs regulate YPP gene expression in the fat body both directly and indirectly. In vitro fat body culture experiments show that $Y P P$ expression is directly induced by the combination of the $20 \mathrm{E}$ and ILP signaling pathways acting together to activate gene expression. Roy et al., demonstrated the synergistic relationship of $20 \mathrm{E}$ and insulin by incubating isolated mosquito fat bodies for $3 \mathrm{~h}$ in different mixtures of porcine insulin and 20E (Roy et al., 2007). These experiments show that neither 20E nor insulin has an effect on expression individually; however a strong synergistic effect occurs when presented together.

ILPs also regulate YPP expression indirectly through the regulation of ecdysone synthesis. A blood meal stimulates the secretion of ILPs amongst other neuropeptides, namely $\mathrm{OEH}$, that regulate ecdysteroid production (Riehle and Brown, 1999; Roy et al., 2007; Brown et al., 2008). In Ae. aegypti, ILP3 is characterized as the ILP responsible for initiating egg development (Brown et al., 2008). An experiment by Brown et al. demonstrates that ILP3, bovine, and porcine insulin stimulate the ovaries to produce ecdysteroids in decapitated females (Brown et al., 2008).

Manipulations of the ILP-signaling pathway in mosquitoes have resulted in profound changes in egg production. RNAi-mediated knockdown of the phosphatidylinositol3,4,5-trisphosphate 3-phosphatase PTEN6, a suppressor of ILP signaling resulted in a significant increase in viable eggs produced by females (Arik et al., 2009).

One downstream effector gene of the ILP-signaling pathway has been characterized in mosquitoes. The forkhead-box transcription factor FOXO is a major target of ILP signaling in insects and vertebrates (Hwangbo et al., 2004). FOXO levels rise in the mosquito fat body after a blood meal and RNAi-mediated knockdown of this factor resulted in abolishment of $v g 1$ gene expression in isolated fat bodies that were stimulated with amino acids. Also, FOXO knockdown mosquitoes produced smaller egg numbers after a blood meal (Hansen et al., 2007). It is not known if this is due to direct or indirect effects.

\section{CROSSTALK BETWEEN PATHWAYS}

The interplay between the different signaling pathways regulating YPP gene expression is complex and only partially understood. However, significant levels of crosstalk appear to occur between the aforementioned signaling pathways in the different organs and tissues of adult female mosquitos. Several studies address these complex interactions.

\section{JH/TOR}

$\mathrm{JH}$ and nutrient signaling are connected in mosquitoes in two distinct ways. A recent study shows that $\mathrm{JH}$ biosynthesis is in part regulated by nutrient signaling via the TOR pathway (PerezHedo et al., 2013). Nutritional information impacts JH levels by regulating the expression of genes associated with the JH synthesis pathway in the corpora allata. A similar regulatory interplay between TOR signaling and $\mathrm{JH}$ expression was also found in the German cockroach, Blatella germanica (Maestro et al., 2009). $\mathrm{JH}$ in turn, regulates the expression of several genes coding for proteins associated with the TOR-signaling machinery in the mosquito fat body (Roy et al., 2007). Presence or absence of TOR pathway components profoundly influences the interpretation of nutritional signals by the fat body after a blood meal and decide if a blood meal results in immediate or delayed YPP synthesis.

\section{ILP3/TOR, ILP3/ECDYSONE, ILP/ECDYSONE/JH}

ILP3 has emerged as a key regulator of vitellogenesis via multiple mechanisms. After a blood meal, ILP3 activates the expression of 
trypsin proteases in the midgut of mosquitoes thereby enhancing blood digestion and indirectly affecting TOR-mediated nutrient signaling (Gulia-Nuss et al., 2011). Three studies have demonstrated the effect of ILP signaling on ecdysone synthesis. The first study showed that unfed mosquitoes receiving ectopic treatment with bovine and porcine insulin results in activation of ecdysteroid secretion (Graf et al., 1997). The second study finds that ILP3 stimulates ecdysone synthesis and YPP uptake in female mosquitoes (Brown et al., 2008). The third study shows that insulin only stimulates ecdysteroid production when the mosquitoes are pre-treated with the $\mathrm{JH}$ analog methoprene (Borovsky et al., 1986).

A study in the red flour beetle, Triboleum castaneum, found that $\mathrm{JH}$ regulates $v g$ gene expression through ILP signaling (Sheng et al., 2011). To our knowledge this has not been tested in mosquitoes, yet.

\section{SYNOPSIS AND OUTLOOK}

Figure 2E shows a schematic of the organization of transcription factor binding sites in the $v g 1$ promoter of Ae. aegypti. Several signaling pathways converge in the regulation of these and other YPP genes. In recent years our understanding of the signaling pathways involved and the interplay between them has undergone rapid growth. We know that at least four distinct pathways are involved: juvenile hormone, ecdysone, insulin, and the TOR nutritional signaling pathway. The challenge for the future is to understand the crosstalk between these pathways in different organs and during different physiological stages in adult female mosquitoes. Based upon the current understanding of signaling in mosquito reproduction we feel that the ILPs may play a key regulatory role in the determination of autogenous vs. anautogenous life histories in mosquitoes.

In addition, while ecdysone- and juvenile hormone analogs are already used as insect growth regulators, a challenge for the future is to use the novel and detailed understanding of nutrient and insulin-signaling pathways to develop new strategies for mosquito control.

\section{ACKNOWLEDGMENTS}

This work was as supported by grants from the NIH/NIGMS to Immo A. Hansen: 1SC2GM092300 and 5SC1AI109055.

\section{REFERENCES}

Arik, A. J., Rasgon, J. L., Quicke, K. M., and Riehle, M. A. (2009). Manipulating insulin signaling to enhance mosquito reproduction. BMC Physiol. 9:15. doi: 10.1186/1472-6793-9-15

Attardo, G. M., Hansen, I. A., and Raikhel, A. S. (2005). Nutritional regulation of vitellogenesis in mosquitoes: implications for anautogeny. Insect Biochem. Mol. Biol. 35, 661-675. doi: 10.1016/j.ibmb.2005.02.013

Attardo, G. M., Hansen, I. A., Shiao, S. H., and Raikhel, A. S. (2006). Identification of two cationic amino acid transporters required for nutritional signaling during mosquito reproduction. J. Exp. Biol. 209(Pt 16), 3071-3078. doi: 10.1242/jeb.02349

Attardo, G. M., Higgs, S., Klingler, K. A., Vanlandingham, D. L., and Raikhel, A. S. (2003). RNA interference-mediated knockdown of a GATA factor reveals a link to anautogeny in the mosquito Aedes aegypti. Proc. Natl. Acad. Sci. USA. 100, 13374-13379. doi: 10.1073/pnas.2235649100

Bai, H., Ramaseshadri, P., and Palli, S. R. (2007). Identification and characterization of juvenile hormone esterase gene from the yellow fever mosquito, Aedes aegypti. Insect Biochem. Mol. Biol. 37, 829-837. doi: 10.1016/j.ibmb.2007.05.010
Borovsky, D., Whisenton, L. R., Thomas, B. R., and Fuchs, M. S. (1986). Biosynthesis and distribution of ecdysone and 20-OH-ecdysone in Aedes aegypti. Arch. Insect Biochem. Physiol. 3, 19-30. doi: 10.1002/arch.940030104

Brown, M. R., and Cao, C. (2001). Distribution of ovary ecdysteroidogenic hormone I in the nervous system and gut of mosquitoes. J. Insect Sci. 1:3. Available online at: ncbi.nlm.nih.gov/pmc/articles/PMC355887/

Brown, M. R., Clark, K. D., Gulia, M., Zhao, Z., Garczynski, S. F., Crim, J. W., et al. (2008). An insulin-like peptide regulates egg maturation and metabolism in the mosquito Aedes aegypti. Proc. Natl. Acad. Sci. U.S.A. 105, 5716-5721. doi: 10.1073/pnas.0800478105

Carpenter, V. K., Drake, L. L., Aguirre, S. E., Price, D. P., Rodriguez, S. D., and Hansen, I. A. (2012). SLC7 amino acid transporters of the yellow fever mosquito Aedes aegypti and their role in fat body TOR signaling and reproduction. J. Insect Physiol. 58, 513-522. doi: 10.1016/j.jinsphys.2012.01.005

Chen, L., Zhu, J. S., Sun, G. Q., and Raikhel, A. S. (2005). The early gene Broad is involved in the ecdysterold hierarchy governing vitellogenesis of the mosquito Aedes aegypti (vol 33, pg 743, 2004). J. Mol. Endocrinol. 34, 262-262. doi: 10.1677/jme.1.01531e

Cho, W. L., Tsao, S. M., Hays, A. R., Walter, R., Chen, J. S., Snigirevskaya, E. S., et al. (1999). Mosquito cathepsin B-like protease involved in embryonic degradation of vitellin is produced as a latent extraovarian precursor. J. Biol. Chem. 274, 13311-13321. doi: 10.1074/jbc.274.19.13311

Clements, A. N. (eds.). (1992). The Biology of Mosquitoes. London: Chapman and Hall.

Clifton, M. E., and Noriega, F. G. (2012). The fate of follicles after a blood meal is dependent on previtellogenic nutrition and juvenile hormone in Aedes aegypti. J. Insect Physiol. 58, 1007-1019. doi: 10.1016/j.jinsphys.2012.05.005

Deitsch, K. W., and Raikhel, A. S. (1993). Cloning and analysis of the locus for mosquito vitellogenic carboxypeptidase. Insect Mol. Biol. 2, 205-213. doi: 10.1111/j.1365-2583.1994.tb00140.x

De Meyts, P. (2004). Insulin and its receptor: structure, function and evolution. Bioessays 26, 1351-1362. doi: 10.1002/bies.20151

Dhara, A., Eum, J. H., Robertson, A., Gulia-Nuss, M., Vogel, K. J., Clark, K. D., et al. (2013). Ovary ecdysteroidogenic hormone functions independently of the insulin receptor in the yellow fever mosquito, Aedes aegypti. Insect Biochem. Mol. Biol. 12, 1100-1108. doi: 10.1016/j.ibmb.2013.09.004

Edgar, K. A., Noriega, F. G., Bonning, B. C., and Wells, M. A. (2000). Recombinant juvenile hormone esterase, an effective tool for modifying juvenile hormonedependent expression of the early trypsin gene in mosquitoes. Insect Mol. Biol. 9, 27-31. doi: 10.1046/j.1365-2583.2000.00154.x

Gade, G., Hoffmann, K. H., and Spring, J. H. (1997). Hormonal regulation in insects: facts, gaps, and future directions. Physiol. Rev. 77, 963-1032.

Gilbert, L. I. (2004). Halloween genes encode P450 enzymes that mediate steroid hormone biosynthesis in Drosophila melanogaster. Mol. Cell. Endocrinol. 215, 1-10. doi: 10.1016/j.mce.2003.11.003

Gilbert, L. I., Rybczynski, R., and Warren, J. T. (2002). Control and biochemical nature of the ecdysteroidogenic pathway. Annu. Rev. Entomol. 47, 883-916. doi: 10.1146/annurev.ento.47.091201.145302

Graf, R., Neuenschwander, S., Brown, M. R., and Ackermann, U. (1997). Insulinmediated secretion of ecdysteroids from mosquito ovaries and molecular cloning of the insulin receptor homologue from ovaries of bloodfed Aedes aegypti. Insect Mol. Biol. 6, 151-163. doi: 10.1111/j.1365-2583.1997.tb00083.x

Gulia-Nuss, M., Robertson, A. E., Brown, M. R., and Strand, M. R. (2011). Insulin-like peptides and the target of rapamycin pathway coordinately regulate blood digestion and egg maturation in the mosquito Aedes aegypti. PLoS ONE 6:e20401. doi: 10.1371/journal.pone.0020401

Hagedorn, H. H. (1982). Ecdysone and the Adult Mosquito. Gen. Comp. Endocrinol. $46,374-375$.

Hagedorn, H. H. (1989). Ecdysone Production by Insect Ovaries - a Citation Classic Commentary on the Ovary as a Source of Alpha-Ecdysone in an Adult Mosquito by Hagedorn,H.H., Oconnor,J.D., Fuchs,M.S., Sage,B., Schlaeger,D.A., and Bohm,M.K. Curr. Cont. Agricult. Biol. Environ. Sci. 13, 14-14.

Hagedorn, H. H., O'Connor, J. D., Fuchs, M. S., Sage, B., Schlaeger, D. A., and Bohm, M. K. (1975). The ovary as a source of alpha-ecdysone in an adult mosquito. Proc. Natl. Acad. Sci. U.S.A. 72, 3255-3259. doi: 10.1073/pnas.72.8.3255

Hall, B. L., and Thummel, C. S. (1998). The RXR homolog Ultraspiracle is an essential component of the Drosophila ecdysone receptor. Development 125, 4709-4717. 
Hansen, I. A., Attardo, G. M., Park, J. H., Peng, Q., and Raikhel, A. S. (2004). Target of rapamycin-mediated amino acid signaling in mosquito anautogeny. Proc. Natl. Acad. Sci. U.S.A. 101, 10626-10631. doi: 10.1073/pnas.0403460101

Hansen, I. A., Attardo, G. M., Roy, S. G., and Raikhel, A. S. (2005). Target of rapamycin-dependent activation of S6 kinase is a central step in the transduction of nutritional signals during egg development in a mosquito. J. Biol. Chem. 280, 20565-20572. doi: 10.1074/jbc.M500712200

Hansen, I. A., Sieglaff, D. H., Munro, J. B., Shiao, S. H., Cruz, J., Lee, I. W., et al. (2007). Forkhead transcription factors regulate mosquito reproduction. Insect Biochem. Mol. Biol. 37, 985-997. doi: 10.1016/j.ibmb.2007.05.008

Hay, N., and Sonenberg, N. (2004). Upstream and downstream of mTOR. Genes Dev. 18, 1926-1945. doi: 10.1101/gad.1212704

Hwangbo, D. S., Gershman, B., Tu, M. P., Palmer, M., and Tatar, M. (2004). Drosophila dFOXO controls lifespan and regulates insulin signalling in brain and fat body. Nature 429, 562-566. doi: 10.1038/nature02549

Jindra, M., Palli, S. R., and Riddiford, L. M. (2013). The juvenile hormone signaling pathway in insect development. Annu. Rev. Entomol. 58, 181-204. doi: 10.1146/annurev-ento-120811-153700

Kapitskaya, M., Wang, S., Cress, D. E., Dhadialla, T. S., and Raikhel, A. S. (1996). The mosquito ultraspiracle homologue, a partner of ecdysteroid receptor heterodimer: cloning and characterization of isoforms expressed during vitellogenesis. Mol. Cell. Endocrinol. 121, 119-132. doi: 10.1016/0303-7207(96)03847-6

Kapitskaya, M. Z., Li, C., Miura, K., Segraves, W., and Raikhel, A. S. (2000). Expression of the early-late gene encoding the nuclear receptor HR3 suggests its involvement in regulating the vitellogenic response to ecdysone in the adult mosquito. Mol. Cell. Endocrinol. 160, 25-37. doi: 10.1016/S03037207(99)00253-1

Lassiter, M. T., Apperson, C. S., Crawford, C. L., and Roe, R. M. (1994). Juvenilehormone metabolism during adult development of culex-quinquefasciatus (Diptera, Culicidae). J. Med. Entomol. 31, 586-593.

Lassiter, M. T., Apperson, C. S., and Roe, R. M. (1995). Juvenile-hormone metabolism during the 4 th stadium and pupal stage of the southern house mosquito, culex-quinquefasciatus say. J. Insect Physiol. 41, 869-876. doi: 10.1016/0022-1910(95)00037-U

Lassiter, M. T., Apperson, C. S., and Roe, R. M. (1996). Juvenile hormone metabolism in the ovary, gut, head and carcass after blood feeding in the southern house mosquito, Culex quinquefasciatus. Comp. Biochem. Physiol. B Biochem. Mol. Biol. 113, 229-237. doi: 10.1016/0305-0491(95)02018-7

Li, C., Kapitskaya, M. Z., Zhu, J., Miura, K., Segraves, W., and Raikhel, A. S. (2000). Conserved molecular mechanism for the stage specificity of the mosquito vitellogenic response to ecdysone. Dev. Biol. 224, 96-110. doi: 10.1006/dbio.2000.9792

Li, M., Mead, E. A., and Zhu, J. (2011). Heterodimer of two bHLH-PAS proteins mediates juvenile hormone-induced gene expression. Proc. Natl. Acad. Sci. U.S.A. 108, 638-643. doi: 10.1073/pnas. 1013914108

Luckhart, S., and Riehle, M. A. (2007). The insulin signaling cascade from nematodes to mammals: insights into innate immunity of Anopheles mosquitoes to malaria parasite infection. Dev. Comp. Immunol. 31, 647-656. doi: 10.1016/j.dci.2006.10.005

Maestro, J. L., Cobo, J., and Belles, X. (2009). Target of rapamycin (TOR) mediates the transduction of nutritional signals into juvenile hormone production. J. Biol. Chem. 284, 5506-5513. doi: 10.1074/jbc.M807042200

Marquardt, W. C. (2005). Biology of Disease Vectors. Burlington, MA: Elsevier Academic press.

Marquardt, W. H. (2004). Biology of Disease Vectors. Burlington, MA: Academic Press.

Martin, D., Piulachs, M. D., and Raikhel, A. S. (2001b). A novel GATA factor transcriptionally represses yolk protein precursor genes in the mosquito Aedes aegypti via interaction with the CtBP corepressor. Mol. Cell. Biol. 21, 164-174. doi: 10.1128/MCB.21.1.164-174.2001

Martin, D., Wang, S. F., and Raikhel, A. S. (2001a). The vitellogenin gene of the mosquito Aedes aegypti is a direct target of ecdysteroid receptor. Mol. Cell. Endocrinol. 173, 75-86. doi: 10.1016/S0303-7207(00)00413-5

Miura, K., Zhu, J., Dittmer, N. T., Chen, L., and Raikhel, A. S. (2002). A COUPTF/Svp homolog is highly expressed during vitellogenesis in the mosquito Aedes aegypti. J. Mol. Endocrinol. 29, 223-238. doi: 10.1677/jme.0.0290223

Nene, V., Wortman, J. R., Lawson, D., Haas, B., Kodira, C., Tu, Z. J., et al. (2007). Genome sequence of Aedes aegypti, a major arbovirus vector. Science 316, 1718-1723. doi: 10.1126/science. 1138878
Noriega, F. G. (2004). Nutritional regulation of JH synthesis: a mechanism to control reproductive maturation in mosquitoes? Insect Biochem. Mol. Biol. 34, 687-693. doi: 10.1016/j.ibmb.2004.03.021

$\mathrm{Ou}, \mathrm{Q}$. X., and King-Jones, K. (2013). What goes up must come down: transcription factors have their say in making ecdysone pulses. Curr. Top. Dev. Biol. 103, 35-71. doi: 10.1016/B978-0-12-385979-2.00002-2

Pakpour, N., Corby-Harris, V., Green, G. P., Smithers, H. M., Cheung, K. W., Riehle, M. A., et al. (2012). Ingested human insulin inhibits the mosquito NFkappaB-dependent immune response to Plasmodium falciparum. Infect. Immun. 80, 2141-2149. doi: 10.1128/IAI.00024-12

Park, J. H., Attardo, G. M., Hansen, I. A., and Raikhel, A. S. (2006). GATA factor translation is the final downstream step in the amino acid/target-of-rapamycinmediated vitellogenin gene expression in the anautogenous mosquito Aedes aegypti. J. Biol. Chem. 281, 11167-11176. doi: 10.1074/jbc.M601517200

Perez-Hedo, M., Rivera-Perez, C., and Noriega, F. G. (2013). The insulin/TOR signal transduction pathway is involved in the nutritional regulation of juvenile hormone synthesis in Aedes aegypti. Insect Biochem. Mol. Biol. 43, 495-500. doi: 10.1016/j.ibmb.2013.03.008

Pondeville, E., Maria, A., Jacques, J. C., Bourgouin, C., and Dauphin-Villemant, C. (2008). Anopheles gambiae males produce and transfer the vitellogenic steroid hormone 20-hydroxyecdysone to females during mating. Proc. Natl. Acad. Sci. U.S.A. 105, 19631-19636. doi: 10.1073/pnas.0809264105

Prestwich, G. D., Wojtasek, H., Lentz, A. J., and Rabinovich, J. M. (1996). Biochemistry of proteins that bind and metabolize juvenile hormones. Arch. Insect Biochem. Physiol. 32, 407-419. doi: 10.1002/(SICI)1520-6327(1996)32:3/4<407::AID-ARCH13>3.0.CO;2-G

Price, D. P., Nagarajan, V., Churbanov, A., Houde, P., Milligan, B., Drake, L. L., et al. (2011). The fat body transcriptomes of the yellow fever mosquito Aedes aegypti, pre- and post-blood meal. PLoS ONE 6:e22573. doi: 10.1371/journal.pone.0022573

Provost-Javier, K. N., Chen, S., and Rasgon, J. L. (2010). Vitellogenin gene expression in autogenous Culex tarsalis. Insect Mol. Biol. 19, 423-429. doi: 10.1111/j.1365-2583.2010.00999.x

Raikhel, A. S., Kokoza, V. A., Zhu, J., Martin, D., Wang, S. F., Li, C., et al. (2002). Molecular biology of mosquito vitellogenesis: from basic studies to genetic engineering of antipathogen immunity. Insect Biochem. Mol. Biol. 32, 1275-1286. doi: 10.1016/S0965-1748(02)00090-5

Raikhel, A. S., Miura, K., and Segraves, W. A. (1999). Nuclear receptors in mosquito vitellogenesis. Am. Zool. 39, 722-735.

Rewitz, K. F., Rybczynski, R., Warren, J. T., and Gilbert, L. I. (2006). The Halloween genes code for cytochrome P450 enzymes mediating synthesis of the insect moulting hormone. Biochem. Soc. Trans. 34, 1256-1260. doi: 10.1042/BST0341256

Rewitz, K. F., Yamanaka, N., Gilbert, L. I., and O'Connor, M. B. (2009). The insect neuropeptide PTTH activates receptor tyrosine kinase torso to initiate metamorphosis. Science 326, 1403-1405. doi: 10.1126/science.1176450

Riddiford, L. M. (2012). How does juvenile hormone control insect metamorphosis and reproduction? Gen. Comp. Endocrinol. 179, 477-484. doi: 10.1016/j.ygcen.2012.06.001

Riehle, M. A., and Brown, M. R. (1999). Insulin stimulates ecdysteroid production through a conserved signaling cascade in the mosquito Aedes aegypti. Insect Biochem. Mol. Biol. 29, 855-860. doi: 10.1016/S0965-1748(99)00084-3

Riehle, M. A., and Brown, M. R. (2002). Insulin receptor expression during development and a reproductive cycle in the ovary of the mosquito Aedes aegypti. Cell Tissue Res. 308, 409-420. doi: 10.1007/s00441-002-0561-8

Riehle, M. A., Fan, Y., Cao, C., and Brown, M. R. (2006). Molecular characterization of insulin-like peptides in the yellow fever mosquito, Aedes aegypti: expression, cellular localization, and phylogeny. Peptides 27, 2547-2560. doi: 10.1016/j.peptides.2006.07.016

Roy, S. G., Hansen, I. A., and Raikhel, A. S. (2007). Effect of insulin and 20hydroxyecdysone in the fat body of the yellow fever mosquito, Aedes aegypti. Insect Biochem. Mol. Biol. 37, 1317-1326. doi: 10.1016/j.ibmb.2007.08.004

Roy, S. G., and Raikhel, A. S. (2011). The small GTPase Rheb is a key component linking amino acid signaling and TOR in the nutritional pathway that controls mosquito egg development. Insect Biochem. Mol. Biol. 41, 62-69. doi: 10.1016/j.ibmb.2010.10.001

Roy, S. G., and Raikhel, A. S. (2012). Nutritional and hormonal regulation of the TOR effector $4 \mathrm{E}$-binding protein (4E-BP) in the mosquito Aedes aegypti. FASEB J. 26, 1334-1342. doi: 10.1096/fj.11-189969 
Sappington, T. W., Kokoza, V. A., Cho, W. L., and Raikhel, A. S. (1996). Molecular characterization of the mosquito vitellogenin receptor reveals unexpected high homology to the Drosophila yolk protein receptor. Proc. Natl. Acad. Sci. U.S.A. 93, 8934-8939. doi: 10.1073/pnas.93.17.8934

Sheng, Z., Xu, J., Bai, H., Zhu, F., and Palli, S. R. (2011). Juvenile hormone regulates vitellogenin gene expression through insulin-like peptide signaling pathway in the red flour beetle, Tribolium castaneum. J. Biol. Chem. 286, 41924-41936. doi: 10.1074/jbc.M111.269845

Shiao, S. H., Hansen, I. A., Zhu, J., Sieglaff, D. H., and Raikhel, A. S. (2008). Juvenile hormone connects larval nutrition with target of rapamycin signaling in the mosquito Aedes aegypti. J. Insect Physiol. 54, 231-239. doi: 10.1016/j.jinsphys.2007.09.007

Spindler, K. D., Honl, C., Tremmel, C., Braun, S., Ruff, H., and Spindler-Barth, M. (2009). Ecdysteroid hormone action. Cell. Mol. Life Sci. 66, 3837-3850. doi: 10.1007/s00018-009-0112-5

Sun, G. Q., Zhu, J. S., and Raikhel, A. S. (2004). The early gene E74B isoform is a transcriptional activator of the ecdysteroid regulatory hierarchy in mosquito vitellogenesis. Mol. Cell. Endocrinol. 218, 95-105. doi: 10.1016/j.mce.2003.12.014

Sun, J., Hiraoka, T., Dittmer, N. T., Cho, K. H., and Raikhel, A. S. (2000). Lipophorin as a yolk protein precursor in the mosquito, Aedes aegypti. Insect Biochem. Mol. Biol. 30, 1161-1171. doi: 10.1016/S0965-1748(00) 00093-X

Tran, H. T., Shaaban, S., Askari, H. B., Walfish, P. G., Raikhel, A. S., and Butt, T. R. (2001). Requirement of co-factors for the ligand-mediated activity of the insect ecdysteroid receptor in yeast. J. Mol. Endocrinol. 27, 191-209. doi: 10.1677/jme.0.0270191

Wang, S. F., Ayer, S., Segraves, W. A., Williams, D. R., and Raikhel, A. S. (2000b). Molecular determinants of differential ligand sensitivities of insect ecdysteroid receptors. Mol. Cell. Biol. 20, 3870-3879. doi: 10.1128/MCB.20.11.38703879.2000

Wang, S. F., Li, C., Sun, G., Zhu, J., and Raikhel, A. S. (2002). Differential expression and regulation by 20-hydroxyecdysone of mosquito ecdysteroid receptor isoforms A and B. Mol. Cell. Endocrinol. 196, 29-42. doi: 10.1016/S03037207(02)00225-3

Wang, S. F., Li, C., Zhu, J., Miura, K., Miksicek, R. J., and Raikhel, A. S. (2000a). Differential expression and regulation by 20-hydroxyecdysone of mosquito ultraspiracle isoforms. Dev. Biol. 218, 99-113. doi: 10.1006/dbio.1999.9575
Wang, S. F., Miura, K., Miksicek, R. J., Segraves, W. A., and Raikhel, A. S. (1998). DNA binding and transactivation characteristics of the mosquito ecdysone receptor-Ultraspiracle complex. J. Biol. Chem. 273, 27531-27540. doi: 10.1074/jbc.273.42.27531

Wu, Q., and Brown, M. R. (2006). Signaling and function of insulinlike peptides in insects. Annu. Rev. Entomol. 51, 1-24. doi: 10.1146/annurev.ento.51.110104.151011

Yao, T. P., Segraves, W. A., Oro, A. E., McKeown, M., and Evans, R. M. (1992). Drosophila ultraspiracle modulates ecdysone receptor function via heterodimer formation. Cell 71, 63-72. doi: 10.1016/0092-8674(92)90266-F

Zhu, J., Miura, K., Chen, L., and Raikhel, A. S. (2000). AHR38, a homolog of NGFI$\mathrm{B}$, inhibits formation of the functional ecdysteroid receptor in the mosquito Aedes aegypti. EMBO J. 19, 253-262. doi: 10.1093/emboj/19.2.253

Zhu, J. S., Chen, L., Sun, G. Q., and Raikhel, A. S. (2006). The competence factor beta Ftz-F1 potentiates ecdysone receptor activity via recruiting a p160/SRC coactivator. Mol. Cell. Biol. 26, 9402-9412. doi: 10.1128/MCB.01318-06

Zou, Z., Saha, T. T., Roy, S., Shin, S. W., Backman, T. W., Girke, T., et al. (2013). Juvenile hormone and its receptor, methoprene-tolerant, control the dynamics of mosquito gene expression. Proc. Natl. Acad. Sci. U.S.A. 110, E2173-2181. doi: $10.1073 /$ pnas. 1305293110

Conflict of Interest Statement: The authors declare that the research was conducted in the absence of any commercial or financial relationships that could be construed as a potential conflict of interest.

Received: 11 January 2014; accepted: 01 March 2014; published online: 20 March 2014. Citation: Hansen IA, Attardo GM, Rodriguez SD and Drake LL (2014) Four-way regulation of mosquito yolk protein precursor genes by juvenile hormone-, ecdysone-, nutrient-, and insulin-like peptide signaling pathways. Front. Physiol. 5:103. doi: 10.3389/fphys.2014.00103

This article was submitted to Invertebrate Physiology, a section of the journal Frontiers in Physiology.

Copyright (c) 2014 Hansen, Attardo, Rodriguez and Drake. This is an open-access article distributed under the terms of the Creative Commons Attribution License (CC BY). The use, distribution or reproduction in other forums is permitted, provided the original author(s) or licensor are credited and that the original publication in this journal is cited, in accordance with accepted academic practice. No use, distribution or reproduction is permitted which does not comply with these terms. 